\title{
Nuclear Data Uncertainty Propagation to Reactivity Coefficients of a Sodium Fast Reactor.
}

\author{
J.J. Herrero, R. Ochoa, J.S. Martínez, C.J. Díez, N. García-Herranz, and O. Cabellos
}

Engineering of innovative reactor concepts requires computational tools capable of producing results with a high level of accuracy. These results are affected by different sources of uncertainty such as the ones coming from nuclear data. The assessment of the uncertainty levels on the design and safety parameters is mandatory.

The uncertainty quantification applied here is based on the adjoint sensitivity analysis. Where the sensitivities of design values to nuclear data are employed together with the nuclear data uncer- tainties to propagate these uncertainties to the design parameters.

The European Sodium Fast Reactor (ESFR) is the innovative reactor model considered here, designed in the framework of a EURATOM Collaborative Project. Some of the relevant safety quantities linked to it are Doppler and void reactivity coefficients, whose uncertainties are quantified. Also the identification of the nuclear reaction data where an improvement will certainly benefit the design accuracy is performed. This work has been performed with the SCALE 6.1 codes suite and its multigroups cross sections library based on ENDF/B-VII.0 evaluation.

\section{INTRODUCTION}

Continuous efforts are being made in the development of Sodium cooled Fast Reactors (SFR) which could have an important role to reach a sustainable, efficient and cost effective nuclear energy production. The EURATOM Collaborative Project for a European Sodium Fast Reactor (CP-ESFR) [1] is dedicated to select pre-conceptual designs for an optimized SFR model.

One of the points of view for optimization is safety enhancement. In this regard, one key parameter is the positive sodium void reactivity insertion following a removal of sodium from the core. The Doppler reactivity insertion, as a result of fuel temperature changes, is also of importance, because its negative value should be addressed. And the reactivity effect driven by material expansions should also be assessed, though it will not be regarded here.

These integral coefficients should be within an uncertainty range that ensures safety. Guiding values for the multiplication factor $k_{\text {eff }}$ and the reactivity coefficients $\rho$, following two recommendations $[2,3]$, are presented in table I.
TABLE I: Target uncertainties on design parameters.

\begin{tabular}{lcc}
\hline \hline & Salvatores & Ishikawa \\
\hline $\mathrm{k}_{\text {eff }}(\% \Delta \mathrm{k} / \mathrm{k})$ & 0.3 & 0.3 \\
Doppler reactivity $(\% \Delta \rho / \rho)$ & 7.0 & 14.0 \\
Na void reactivity $(\% \Delta \rho / \rho)$ & 7.0 & 20.0 \\
\hline \hline
\end{tabular}

The uncertainties due to cross section data for these integral values have been studied. It could be affirmed that the multiplication factor is quite close to the design requirements [4], while reactivity coefficients are still far from desired levels for the void effect [5].

In the following, we present a calculation procedure applied to obtain the uncertainty in sodium void worth and Doppler reactivity for an optimized design of a 3600 MWth SFR based on oxide fuel and proposed in the ESFR Project [6]. Computations have been performed with SCALE 6.1 [7] for cross sections uncertainty propagation to $k_{\text {eff }}$ and $\rho$. Results will be presented together with their uncertainties to evaluate its compliance with the previous limits and to point out the main contributors to such uncertainties. 


\section{EUROPEAN SODIUM FAST REACTOR MODEL}

The selected ESFR core model for this study contains oxide fuel distributed in an inner and an outer region of sub-assemblies (SA). The outer region presents a slightly higher Plutonium content in order to flatten the power shape at the end of the cycle. No minor actinides were charged into the core.

The active region has a flattened shape of about 4.7 meters in diameter by 1.0 meter height of MOX pellets. Above the active core, there is a fission gas plenum filled with helium, followed by a sodium plenum and an absorber layer, covered by and axial shielding section. Below the active region, there is a short fertile blanket formed by UOX pellets, followed by a long fission gas plenum. The sodium plenum, absorber layer and fertile blanket have been added to the original configuration of the CP-ESFR as a result of an optimization effort to reduce the sodium void effect [8].

In the radial direction, there exist five different types of SA: two fuel SA with a different isotopic enrichment, two control elements with different boron carbide compositions and one radial reflector assembly.

Calculations will be performed at nominal conditions with the fuel at $1500 \mathrm{~K}$. In order to compute the reactivities, two different perturbations will be introduced:

- Heating of the active fuel region (fertile blanket not included) to $2573 \mathrm{~K}$.

- Voiding sodium regions in the most unfavorable but realistic way.

The voiding of the core is performed only in the sodium region surrounding each fuel pin from the fertile blanket to the top of the core. Keeping the sodium under the fertile blanket region and between fuel SA wrappers increases the flux level but avoids leakages, maximizing reactivity insertion. On the other side, the sodium in the upper plenum is removed as this should be closer to reality during a possible accident and benefits from the optimized configuration advantages.

\section{UNCERTAINTY PROPAGATION TO REACTIVITY COEFFICIENTS}

The study of reactivity coefficients for fast reactors is usually performed through perturbation theory to obtain insight their dependence on the different cross sections, isotopes and regions of the core [9]. The reactivity coefficients assessment is strongly affected by nuclear data uncertainty, and our aim here is to quantify such effect.

A full core 3D model has been prepared following the project specifications to compute forward and adjoint flux distributions for each considered state with the Monte Carlo code KENO-VI from the SCALE 6.1 package. These solutions are used in the TSUNAMI module to compute sensitivities $S_{k}$ of $\mathrm{k}_{\mathrm{eff}}$ to cross sections data using perturbation theory.

Next, the TSAR module combines these sensitivities employing Eq. (1) to obtain the sensitivities for the reactivity $S_{\rho}$, which is a function of the multiplication factors for the nominal and the perturbed states, $k_{1}$ and $k_{2}$ respectively [10].

$S_{\rho}=\frac{S_{k_{1}} / k_{1}-S_{k_{2}} / k_{2}}{\rho} ; \rho=\frac{k_{2}-k_{1}}{k_{1} k_{2}}$

Finally, these sensitivities are combined with cross sections uncertainty data to obtain a final evaluation of the impact of that uncertainty on the reactivity coefficient.

The 238 energy groups cross sections library employed is based on ENDF/B-VII.0 11]. The uncertainty information is compiled in a 44 energy groups structure in SCALE 6.1.

\section{RESULTS}

Calculations were performed for the three different states already mentioned, i.e. nominal, heated and voided. In table II the corresponding $\mathrm{k}_{\mathrm{eff}}$ and their relative uncertainty $\Delta k / k$ due to cross section uncertainties are included, all quantities with their corresponding stochastic uncertainty.

TABLE II: $\mathrm{k}_{\mathrm{eff}}$ values and propagated uncertainties for each state. Stochastics uncertainties are included.

\begin{tabular}{lrrr}
\hline & Nominal & Heated & Voided \\
\hline $\mathrm{k}_{\text {eff forward }}$ & $1.02110 \pm 10^{-4}$ & $1.01593 \pm 10^{-4}$ & $1.02528 \pm 10^{-4}$ \\
$\mathrm{k}_{\text {eff }}$ adjoint & $1.02128 \pm 10^{-3}$ & $1.01531 \pm 10^{-3}$ & $1.02502 \pm 10^{-3}$ \\
$\% \Delta k / k$ & $1.589 \pm 10^{-3}$ & $1.604 \pm 10^{-3}$ & $1.672 \pm 10^{-3}$ \\
\hline \hline
\end{tabular}

Table III presents the reactivity values and their uncertainties from the nuclear data, accompanied also by their statistical uncertainties from the Monte Carlo method. Here, the uncertainty in the voiding reactivity over 100 $\mathrm{pcm}$ is well above the desired target accuracy of $7 \%$, and still above the more relaxed limit of $20 \%$ contained in table I

TABLE III: Reactivity and propagated uncertainty for perturbed states. Statistical uncertainty is included.

\begin{tabular}{lcc}
\hline \hline & Fuel Doppler Sodium void \\
\hline$\rho(\mathrm{pcm})$ & $-499 \pm 14$ & $399 \pm 14$ \\
$\% \Delta \rho / \rho$ & $5.8 \pm 0.4$ & $30.6 \pm 0.4$ \\
\hline \hline
\end{tabular}

The main contributors to the uncertainty in the Doppler reactivity effect are listed in table IV , where $\bar{\nu}$ 
is the average total fission neutrons emitted and $\chi$ is the fission spectrum. Now not only ${ }^{238} \mathrm{U}$ and ${ }^{239} \mathrm{Pu}$ reactions are present as it usually happens for multiplication factors, but also the elastic scattering reactions with ${ }^{23} \mathrm{Na}$ and ${ }^{16} \mathrm{O}$ are of importance. This stresses the fact that the dependencies of the reactivity coefficients on the neutronic parameters can be notably different from the dependencies of the multiplication constants of each state.

TABLE IV: Main uncertainty contributions for Doppler heating reactivity with their statistical uncertainty.

\begin{tabular}{llcc}
\hline \hline \multicolumn{2}{c}{ Reaction } & $\% \Delta \mathrm{k} / \mathrm{k}$ & Std.Dev.(\%) \\
\hline${ }^{238} \mathrm{U}$ & $\left(n, n^{\prime}\right)$ & 4.78 & 7.25 \\
${ }^{16} \mathrm{O}$ & $(n, n)$ & 1.53 & 7.17 \\
${ }^{23} \mathrm{Na}$ & $(n, n)$ & 1.46 & 5.71 \\
${ }^{239} \mathrm{Pu}$ & $\chi$ & 0.98 & 0.07 \\
${ }^{238} \mathrm{U}$ & $(n, n)$ & 0.88 & 0.14 \\
${ }^{239} \mathrm{Pu}$ & $(n, \gamma)$ & 0.85 & 0.30 \\
${ }^{239} \mathrm{Pu}$ & $\bar{\nu}$ & 0.83 & 0.04 \\
\hline \hline
\end{tabular}

The same observation is valid for the void reactivity effect as shown in table $\mathrm{V}$, where now ${ }^{238} \mathrm{U}$ and ${ }^{23} \mathrm{Na}$ elastic and inelastic scatterings are the main focus.

TABLE V: Main uncertainty contributions for Na voiding reactivity with their statistical uncertainty.

\begin{tabular}{llcc}
\hline \hline \multicolumn{2}{c}{ Reaction } & $\% \Delta \mathrm{k} / \mathrm{k}$ & Std.Dev.(\%) \\
\hline${ }^{238} \mathrm{U}$ & $\left(n, n^{\prime}\right)$ & 21.9 & 1.65 \\
${ }^{23} \mathrm{Na}$ & $\left(n, n^{\prime}\right)$ & 12.6 & 0.17 \\
${ }^{238} \mathrm{U}$ & $(n, n) \cdot\left(n, n^{\prime}\right)$ & 7.47 & 0.43 \\
${ }^{23} \mathrm{Na}$ & $(n, n)$ & 7.47 & 0.98 \\
${ }^{238} \mathrm{U}$ & $(n, \gamma)$ & 7.43 & 0.10 \\
${ }^{239} \mathrm{Pu}$ & $\bar{\nu}$ & 7.05 & 0.01 \\
${ }^{239} \mathrm{Pu}$ & $(n, f)$ & 4.28 & 0.01 \\
\hline \hline
\end{tabular}

The reactions appearing as the main contributors to the uncertainty are in agreement with other recent indications [12]. In contrast to $\mathrm{k}_{\text {eff }}$ calculations, in the case of reactivity computations, importance of the uncertainty in $(n, n)$ reactions with ${ }^{238} \mathrm{U}$ and ${ }^{23} \mathrm{Na}$ should be stressed. Together with the possible need of good correlated uncertainty data between reactions as indicates the appearance of the ${ }^{238} \mathrm{U}(n, n)-\left(n, n^{\prime}\right)$ covariance term.

\section{CONCLUSIONS}

The contribution of cross sections uncertainties to reactivity coefficients for heated and voided perturbed states of an oxide core design of the European Sodium Fast Reactor model have been quantified with the SCALE 6.1 codes suite.

Considering current target accuracies for these parameters in the engineering design phase, the Doppler reactivity coefficient is inside the requested limit, while the sodium void reactivity coefficient uncertainty is outside the desired boundaries.

Additionally, the main isotope-reaction pairs responsible for the uncertainty values are indicated as objectives for a future uncertainty reduction in the nuclear data evaluations. Particularly, elastic and inelastic scattering cross sections for ${ }^{238} \mathrm{U}$ and ${ }^{23} \mathrm{Na}$ are pointed out.

The research leading to these results has received funding from the European Atomic Energy Community's 7th Framework Program in the ANDES project under grant agreement No. 249671. It has also been partially funded by the specific collaborative agreement between CIEMAT and UNED/UPM in the area of High level waste transmutation. The core specifications used for this analysis come from the ESFR Collaborative Project, from the European Community's Seventh Framework Program [FP7/20072013].

[1] G. L. Fiorini and A. Vasile, Nucl. Eng. Design 241, 3461 (2011)

[2] M. Ishikawa, Nucl. Data Sheets 109, 2778 (2008)

[3] M. Salvatores, G. Palmiotti, G. Aliberti, H. Hiruta, et al., Nucl. Data Sheets 109, 2725 (2008)

[4] J. L. Rowlands, Annals Nuc. Energy 37, 1666 (2010)

[5] J. Tommasi, P. Archier, and J. M. Ruggieri, Annals Nucl. Energy 37, 1534 (2010)

[6] D. Blanchet, ESFR Working Horses Core Concept Definition, Tech. Rep. (CP-ESFR, 2009)

[7] S. M. Bowman, Nucl. Tech. 174, 126 (2011)
[8] A. Rineiski et al., Synthesis of options to optimize feedback coefficients, Tech. Rep. (CP-ESFR, 2011)

[9] K. Sun, J. Krepel, K. Mikityuk, et al., Annals Nucl. Energy 38, 1645 (2011)

[10] M. L. Williams, Nucl. Science Eng. 155, 18 (2007)

[11] M. B. Chadwick, P. Oblozinsky, M. Herman, N. M. Greene, et al., Nucl. Data Sheets 107, 2931 (2006)

[12] G. Palmiotti and M. Salvatores, Jour. Nucl. Science Tech. 48, $612(2011)$ 